\title{
Detection of Glaucoma and Other Vision-Threatening Ocular Diseases in the Population Recruited at Specific Health Checkups in Japan
}

This article was published in the following Dove Press journal:

Clinical Epidemiology

\section{Masakazu Yamada (D) \\ Yoshimune Hiratsuka (iD ${ }^{2}$ \\ Tadashi Nakano $\mathbb{D D}^{3}$ \\ Yoshiyuki Kita (D) \\ Tomoyuki Watanabe (D) ${ }^{3}$ \\ Hiroshi Tamura $\mathbb{I D}^{4,5}$ \\ Ryo Kawasaki ${ }^{6}$ \\ Tetsuji Yokoyama ${ }^{7}{ }^{7}$ \\ Shigeru Takano ${ }^{8}$}

'Department of Ophthalmology, Kyorin University School of Medicine, Mitaka, Japan; ${ }^{2}$ Department of Ophthalmology, Juntendo University School of Medicine, Tokyo, Japan; ${ }^{3}$ Department of Ophthalmology, The Jikei University School of Medicine, Tokyo, Japan; ${ }^{4}$ Department of Ophthalmology and Visual Sciences, Kyoto University Graduate School of Medicine, Kyoto, Japan; ${ }^{5}$ Center for Innovative Research and Education in Data Science, Institute for Liberal Arts and Sciences, Kyoto University, Kyoto, Japan; ${ }^{6}$ Artificial Intelligence Center for Medical Research and Application, Osaka University Hospital, Suita, Japan; ${ }^{7}$ Department of Health Promotion, National Institute of Public Health, Wako, Japan; ${ }^{8}$ Japan Ophthalmologist Association, Tokyo, Japan
Correspondence: Masakazu Yamada Department of Ophthalmology, Kyorin University School of Medicine, Mitaka, Japan

Email yamadamasakazu@ks.kyorin-u.ac.jp
Background: The efficacy of vision screening for adults has not been well established. The present study aimed to investigate the prevalence of vision-threatening ocular diseases, including glaucoma, among subjects who participated in specific health checkups in Japan. Methods: This cross-sectional study included 1360 individuals who underwent comprehensive ophthalmic examinations at 16 ophthalmology clinics located in three municipalities We surveyed the study participants using a questionnaire. The participants also underwent visual acuity and refraction tests, intraocular pressure tests, slit-lamp microscopy, fundus examinations, fundus photography, optical coherence tomography, and static perimetry.

Results: The mean age of the subjects was $63.7 \pm 8.7$ years (range, $40-74$ years). Among the 1360 participants, $168(12.4 \%)$ were diagnosed with glaucoma and $33(2.4 \%)$ with preperimetric glaucoma. Cataracts were seen in 741 participants $(54.5 \%)$, and $77(5.7 \%)$ were diagnosed with clinically significant cataracts. Retinal diseases included macular degeneration $(1.2 \%)$, diabetic retinopathy $(1.0 \%)$, chorioretinal atrophy $(0.5 \%)$, macular epiretinal membrane $(2.9 \%)$, branch retinal vein occlusion $(0.7 \%)$, and others $(2.0 \%)$. Regarding the type of glaucoma, $93.5 \%$ of participants with glaucoma were diagnosed with open-angle glaucoma in a broad sense $(81.0 \%$ with normal-tension glaucoma and $12.5 \%$ with primary open-angle glaucoma). Multivariate analysis showed that male sex, age, systemic comorbidities, and myopia were significant risk factors for open-angle glaucoma.

Conclusion: Many adults with ocular diseases were screened by ophthalmic checkups. The addition of simultaneous ophthalmic checkups to specific health checkups could be an effective measure for the prevention of visual impairment in the older population.

Keywords: cataract, glaucoma, ocular disease, prevalence, vision screening

\section{Introduction}

In developed countries, including Japan, visual impairment is an important issue among older adults. ${ }^{1-6}$ The number of visually impaired persons in Japan was estimated to be 1.64 million in 2007 and is expected to reach 2 million by 2030 due to the aging of the population. 5,6 The leading cause of visual impairment was glaucoma, followed by diabetic retinopathy, degenerative myopia, and age-related macular degeneration. ${ }^{5}$ The common features of these ocular diseases are that they are age-related with relatively slow progression and that their treatment modalities 
are limited to maintaining visual function at the most because of their intractable nature.

We have reported that vision screening for adults can be an effective measure for the early detection and treatment of vision-threatening ocular diseases. ${ }^{7-9}$ Glaucoma, the leading cause of blindness in Japan, appears to be a particularly good candidate for vision screening. 5,6 Glaucoma meets many of the conditions that should make it eligible for screening (Wilson's criteria): ${ }^{10}$ it has high prevalence, it is the leading cause of blindness in Japan, early detection is difficult owing to poor subjective symptoms in the early stage, it is a chronic progressive condition with a long duration from onset to visual impairment, and its progression can be suppressed by medical intervention. ${ }^{11}$

However, there is no nationwide screening program for glaucoma and other vision-threatening ocular diseases in Japan. Only a small number of municipalities currently offer vision screening for adults. Each municipality conducts checkups without a standardized protocol. According to previous reports from Japan, ${ }^{9}$ the detection rate of vision screening programs for glaucoma was about $1.9 \%$ to $3.7 \%$, which was less than the estimated prevalence of glaucoma in the Tajimi study ( $5 \%$ in those aged 40 years or older), a representative epidemiological study of glaucoma in Japan. ${ }^{12-14}$ These results indicate insufficient sensitivity of the current screening approach. To reduce visual impairment due to glaucoma, an efficient approach using the existing general health checkup framework may be useful.

Specific health checkups, which are an annual health screening program introduced in 2008 by the Japanese Ministry of Health, Labor and Welfare, are the most common medical checkups in Japan. The target group of the program is all individuals between 40 and 74 years of age, and the national participation rate for specific health checkups in 2017 was $53.1 \%{ }^{15}$ The implementation criteria for ocular fundus examination have changed significantly since the introduction of specific health checkups because the aim of the program is the early detection of individuals at high risk for metabolic syndrome to enable healthcare intervention. Currently, only $13.4 \%$ of those receiving specific health checkups are having ophthalmic checkups. ${ }^{9,16}$ A recent study suggests that additional simultaneous ophthalmic checkups could increase the rate of participation in specific health checkups, and effective use of the existing framework for specific health checkups could be an important measure for protecting eye health nationwide. ${ }^{16}$ However, there have been no detailed reports on the characteristics of glaucoma patients in the population undergoing specific health checkups.

In this study, we conducted detailed comprehensive ophthalmic examinations on persons who underwent specific health checkups. The examinations included optical coherence tomography (OCT) and static perimetry in addition to general ophthalmic examinations in order to make a definitive diagnosis of glaucoma. We report here the characteristics of glaucoma and other vision-threatening ocular diseases among persons who received specific health checkups.

\section{Methods}

\section{Ethics}

This study was conducted according to the Declaration of Helsinki and was performed in accordance with the Ethical Guidelines for Medical and Health Research Involving Human Subjects. The protocol of the study was reviewed and approved by the Institutional Review Board of Kyorin University School of Medicine (No. 1034), and the Research Ethics Committee of the Shinanozaka Clinic, Medical Corporation Shinanokai (No. 2017-22). The subjects received a full explanation of the procedures and provided informed consent for participation prior to inclusion in the study.

\section{Study Population}

In this cross-sectional study, data were collected from 16 ophthalmology clinics located in three municipalities in Japan (Matsue City, Shimane Prefecture; Sendai City, Miyagi Prefecture; and Setagaya Ward, Tokyo Metropolitan). These three municipalities provide additional simultaneous ophthalmic checkups at the same time as specific health checkups from their own supplementary budget. All candidates with national health insurance are eligible for fundus examination as part of the specific health checkups, whether they meet certain criteria or not. Subjects who visited a participating facility to take the opportunity for specific health checkups between June and December 2017 were invited to participate in the study. The participating facilities are listed in the Acknowledgements section.

Background information on the study participants was collected with a questionnaire that contained the following items: sex, age, history of systemic comorbidities (hypertension, diabetes, and other systemic comorbidities), 
history of ophthalmic diseases and surgery, and presence or absence of ophthalmic diseases that were under treatment at the time of the study.

A general eye examination consisting of visual acuity, refraction, intraocular pressure with a noncontact tonometer, slit-lamp microscopy examination, and fundus examination with pupil dilation was conducted. Digital color photographs of the fundus were obtained by the fundus camera. The circumpapillary retinal nerve fiber layer (RNFL) was analyzed by OCT in each facility. As examinations for a definitive diagnosis of glaucoma, static perimetry tests were performed using the Humphrey Visual Field Analyzer (Carl Zeiss Meditec, Oberkochen, Germany) with the SITA Standard 24-2 algorithm.

The ophthalmologist in charge of the examination determined the presence of abnormalities and made a diagnosis of vision-threatening ocular diseases. For glaucoma and preperimetric glaucoma, the examination results were reviewed by a central committee consisting of three glaucoma specialists to ensure a definitive diagnosis. According to the Japan Glaucoma Society, glaucoma is defined as a disease with characteristic changes in the optic nerve and visual field and with functional and structural abnormalities of the eye. ${ }^{11}$ The diagnosis of glaucoma was made based on morphological abnormalities of the optic disc determined by fundus photograph, ${ }^{17}$ the results of RNFL analysis in OCT, and the visual field defect criteria of Anderson and Patella. ${ }^{18}$

\section{Statistical Analysis}

Data for all parameters are presented as mean \pm standard deviation or percentage values. The chi-square test was used to compare percentages between groups. Odds ratios and $95 \%$ confidence intervals were estimated using a univariate or multivariate logistic regression model. Statistical analyses were performed using JMP software, version 10.0.2 (SAS Institute, Cary, NC, USA) or Prism 7 for Mac OS X (GraphPad Software, La Jolla, CA, USA). A $p$-value less than 0.05 was considered to indicate statistical significance.

\section{Results}

\section{Characteristics of the Study Population}

In this study, 1478 participants were initially enrolled. However, 118 patients were excluded due to disqualification or missing data. Therefore, 1360 participants (442 men and 918 women) were included in the final analysis.
In 36 participants (2.6\%), a fundus examination at an ophthalmology clinic was ordered as a detailed medical examination item in the specific health checkup, and an ophthalmological examination was offered to the remaining 1324 participants $(97.4 \%)$ by the municipalities as an optional examination.

The age of the participants was $63.7 \pm 8.7$ years (mean \pm standard deviation), ranging from 40 to 74 years, which was the age range for eligibility for the specific health checkups. With regard to comorbid systemic diseases, 422 participants (31.0\%) had hypertension, $108(7.9 \%)$ had diabetes, and 186 (13.7\%) had other systemic comorbidities, such as cancer, heart disease, and chronic obstructive pulmonary disease.

\section{Glaucoma and Other Chronic Eye Diseases}

Based on comprehensive ophthalmic examinations, glaucoma was diagnosed in 168 participants (12.4\%), and preperimetric glaucoma was diagnosed in 33 participants $(2.4 \%)$ (Table 1$)$. Concerning the quality of ophthalmic examinations, fundus photographs with good quality were obtained from $88.7 \%$ of subjects, and $91.6 \%$ met the

Table I Results of Comprehensive Ophthalmic Examinations

\begin{tabular}{|c|c|c|c|}
\hline Diagnosis & $\begin{array}{l}\text { Number of } \\
\text { Cases }\end{array}$ & $\begin{array}{l}\text { Prevalence } \\
\text { (\%) }\end{array}$ & $\begin{array}{l}95 \% \mathrm{Cl} \\
(\%)\end{array}$ \\
\hline $\begin{array}{l}\text { Glaucoma } \\
\text { Glaucoma (all) } \\
\text { Preperimetric } \\
\text { glaucoma }\end{array}$ & $\begin{array}{l}168 \\
33\end{array}$ & $\begin{array}{l}12.4 \\
2.4\end{array}$ & $\begin{array}{l}10.7-14.2 \\
1.7-3.4\end{array}$ \\
\hline $\begin{array}{l}\text { Cataract } \\
\text { Cataract (any) } \\
\text { Clinically } \\
\text { significant }\end{array}$ & $\begin{array}{l}74 I \\
77\end{array}$ & $\begin{array}{l}54.5 \\
5.7\end{array}$ & $\begin{array}{l}51.8-57.1 \\
4.6-5.7\end{array}$ \\
\hline $\begin{array}{l}\text { Retinal diseases } \\
\text { Epiretinal } \\
\text { membrane }\end{array}$ & 39 & 2.9 & $2.1-3.9$ \\
\hline $\begin{array}{l}\text { Macular } \\
\text { degeneration }\end{array}$ & 16 & 1.2 & $0.7-1.9$ \\
\hline $\begin{array}{l}\text { Diabetic } \\
\text { retinopathy }\end{array}$ & 13 & 1.0 & $0.6-1.6$ \\
\hline $\begin{array}{l}\text { Branch vein } \\
\text { occlusion }\end{array}$ & 10 & 0.7 & $0.4-1.3$ \\
\hline $\begin{array}{l}\text { Chorioretinal } \\
\text { atrophy }\end{array}$ & 7 & 0.5 & $0.3-1.1$ \\
\hline Others & 27 & 2.0 & $1.4-2.9$ \\
\hline
\end{tabular}

Abbreviation: $\mathrm{Cl}$, confidence interval. 
criteria for signal strength of OCT. The comments from ophthalmologist in charge of the examination assisted the judge of central committee in some cases.

Cataracts were noted in 741 participants (54.5\%); however, only 77 participants $(5.7 \%)$ were diagnosed with clinically significant cataracts. In addition, retinal diseases, such as macular epiretinal membrane, macular degeneration, and diabetic retinopathy, were detected.

Table 2 shows the age- and sex-specific prevalence of glaucoma. The prevalence in men was higher than that in women in all age groups except for the forties age group. The prevalence of glaucoma had a trend to increase with age.

Of the 168 participants diagnosed with glaucoma, 37 (22.0\%) had previously been diagnosed with glaucoma and 131 (78.0\%) were newly diagnosed with glaucoma. With regard to the type of glaucoma, 136 participants (81.0\%) had normal-tension glaucoma, 21 (12.5\%) had primary open-angle glaucoma, $5(3.0 \%)$ had primary angle-closure glaucoma, and $6(3.5 \%)$ had other glaucoma conditions. Thus, $93.5 \%$ of participants with glaucoma were diagnosed with open-angle glaucoma in a broad sense (either normal-tension glaucoma or primary openangle glaucoma). According to the mean deviation (MD) of the worse eye for the severity of glaucoma, 128 participants (76.2\%) had MD values $>-6 \mathrm{~dB}, 30(17.9 \%)$ had MD values of -6 to $-12 \mathrm{~dB}, 8(4.8 \%)$ had $\mathrm{MD}$ values of -12 to $-25 \mathrm{~dB}$, and $2(1.2 \%)$ had MD values $<-25 \mathrm{~dB}$.

Regarding open-angle glaucoma in a broad sense, univariate analysis revealed that male sex, hypertension, diabetes, systemic comorbidities (other than hypertension and

Table 2 Age-Specific Prevalence of Glaucoma

\begin{tabular}{|l|l|l|l|}
\hline $\begin{array}{l}\text { Age } \\
\text { Group } \\
\text { (Years) }\end{array}$ & $\begin{array}{l}\text { Men (Cases/ } \\
\text { All) }(95 \% \mathrm{Cl})\end{array}$ & $\begin{array}{l}\text { Women } \\
(\text { Cases/AII) } \\
\%(95 \% \mathrm{CI})\end{array}$ & $\begin{array}{l}\text { Total (Cases/ } \\
\text { All) } \\
\%(95 \% \mathrm{CI})\end{array}$ \\
\hline $40-49$ & $\begin{array}{l}7 / 60 \\
11.7 \%(5.8-22.2)\end{array}$ & $\begin{array}{l}9 / 94 \\
9.6 \%(5.1-17.2)\end{array}$ & $\begin{array}{l}16 / 154 \\
10.4 \%(6.5-16.2)\end{array}$ \\
\hline $50-59$ & $10 / 63$ & $9 / 133$ & $19 / 196$ \\
& $15.9(8.9-26.8)$ & $6.8(3.6-12.4)$ & $9.7(6.3-14.6)$ \\
\hline $60-69$ & $32 / 193$ & $40 / 426$ & $72 / 619$ \\
& $16.6(12.0-22.5)$ & $9.4(7.0-12.5)$ & $11.6(9.3-14.4)$ \\
\hline $70-74$ & $28 / 126$ & $33 / 265$ & $61 / 391$ \\
& $22.2(15.8-30.2)$ & $12.5(9.0-17.0)$ & $15.6(12.3-19.5)$ \\
\hline All subjects & $\begin{array}{l}77 / 442 \\
17.4(14.2-21.2)\end{array}$ & $\begin{array}{l}91 / 918 \\
9.9(8.1-12.0)\end{array}$ & $\begin{array}{l}168 / 1360 \\
12.4(10.7-14.2)\end{array}$ \\
\hline
\end{tabular}

Abbreviation: $\mathrm{Cl}$, confidence interval.
Table 3 Presence or Absence of Open-Angle Glaucoma and Patient Background and Eye Examination Findings in Univariate Analysis

\begin{tabular}{|l|l|l|}
\hline Factor & $\begin{array}{l}\text { Odds } \\
\text { Ratio }\end{array}$ & $\begin{array}{l}\text { 95\% Confidence } \\
\text { Interval }\end{array}$ \\
\hline Sex (male/female) & 1.56 & $1.15-2.12$ \\
Age (per + I year old) & 1.07 & $0.93-2.05$ \\
Hypertension (yes/no) & 1.43 & $1.02-1.98$ \\
Diabetes (yes/no) & 2.06 & $1.24-3.34$ \\
Other systemic comorbidities & 1.96 & $1.32-2.94$ \\
(yes/no) & & \\
Intraocular pressure (per + I & 1.12 & $0.8 \mathrm{I}-1.56$ \\
mmHg) & 1.29 & $1.09-1.49$ \\
Myopia (per - I diopter) & & \\
\hline
\end{tabular}

Table 4 Presence or Absence of Open-Angle Glaucoma and Patient Background and Eye Examination Findings in Multivariate Analysis

\begin{tabular}{|l|l|l|}
\hline Factor & $\begin{array}{l}\text { Adjusted } \\
\text { Odds Ratio }\end{array}$ & $\begin{array}{l}\text { 95\% Confidence } \\
\text { Interval }\end{array}$ \\
\hline Sex (male/female) & 1.85 & $1.33-2.63$ \\
Age (per + I year old) & 1.03 & $1.01-1.05$ \\
Hypertension (yes/no) & 1.19 & $0.83-1.70$ \\
Diabetes (yes/no) & 1.61 & $0.96-2.71$ \\
Other systemic & 1.84 & $1.21-2.79$ \\
comorbidities (yes/no) & & $0.96-1.09$ \\
Intraocular pressure (per + & 1.02 & $1.06-1.09$ \\
I mmHg) & & 1.12 \\
Myopia (per - I diopter) & & \\
\hline
\end{tabular}

diabetes), and myopia had significantly high odds ratios for glaucoma (Table 3). There were no significant differences in the odds ratio for glaucoma according to age and intraocular pressure. Table 4 shows the results of multivariate logistic regression analysis. Male sex, age, systemic comorbidities (other than hypertension and diabetes), and myopia were shown to be significant risk factors for glaucoma.

\section{Discussion}

In this study, we conducted comprehensive detailed ophthalmic examinations in adults aged 40 to 74 years who visited an ophthalmology clinic to take the opportunity for specific health checkups. This study was unique in that static perimetry, a detailed examination for the definitive diagnosis of glaucoma was performed in all participants. In screening programs, detailed examinations are 
not usually conducted for all study subjects. The visual field test is no exception. This is because static perimetry is time-consuming and requires expensive equipment and specialized examiners and therefore is not practical as a screening approach. However, by using static perimetry and OCT in this study, we were able to determine the prevalence and severity of glaucoma in the study population.

Among the 1360 participants registered, the prevalence of glaucoma and preperimetric glaucoma was $12.4 \%$ and $2.4 \%$, respectively, values higher than those reported in previous epidemiological studies in Japan. The higher accuracy of glaucoma diagnosis in this study could be partially explained by the use of static perimetry and OCT for all participants, procedures that were not performed in previous epidemiological studies. ${ }^{12-14,19,20}$ The second reason might be the age of the participants in our study because aging is a well-known risk factor for glaucoma. ${ }^{14}$ Although the age of eligibility for specific health checkups is 40 to 74 years, there were few participants in their 40s and 50s and more in their 60s and 70s. The average age of the participants was $63.7 \pm 8.7$ years, and $74.5 \%$ of the participants were 60 years old or older. When the data in our study were adjusted by the population composition of Japan, the prevalence of glaucoma was estimated to be $11.2 \%$. Therefore, the high prevalence of glaucoma in our study could be explained by the population composition of the participants. The third, myopia was shown to be significant risk factors for glaucoma, which is in good accordance with the previous study in Japan. ${ }^{14}$ However, it is sometimes difficult to differentiate between myopic optic neuropathy and glaucoma. Our study is a cross-sectional survey, and accordingly it is impossible to make a definitive diagnosis by demonstrating glaucomatous progression.

In this study, the prevalence of glaucoma was higher in men than in women. Taking into consideration that $67.5 \%$ of the participants were women, there may have been more male participants who worried about their systemic or ophthalmic health, which may have resulted in the higher prevalence of glaucoma among male participants. However, there were no differences between men and women in age or the proportion of hypertension and systemic comorbidities, although there was a difference in the proportion of diabetes $(12.9 \%$ for men and $5.6 \%$ for women). There were no differences between men and women in the proportion of subjects who had previously been diagnosed with glaucoma or other ocular diseases.
The reasons for the higher prevalence of glaucoma in men remain to be explored.

The rates of early, moderate, and severe glaucoma were $79.5 \%, 16.4 \%$, and $4.1 \%$, respectively. The primary glaucoma types were normal-tension glaucoma $(81.0 \%)$ and open-angle glaucoma (12.5\%), which together accounted for more than $90 \%$ of cases. These findings are consistent with those of previous epidemiological studies in Japan. $^{12-14}$ Only $21 \%$ of participants had been previously diagnosed with glaucoma and were receiving medical care before the comprehensive ophthalmic examination, which supports previous findings that in many patients, glaucoma remains undetected and undiagnosed. Our study confirmed that there are many adults with glaucoma who should be screened by ophthalmic checkups, although the effectiveness of screening programs for glaucoma is controversial. $^{21,22}$ The results of this study are based on real-world data on the general population and reflect the current situation of eye health status of the target population for the specific health checkups in Japan.

Regarding vision-threatening ocular diseases other than glaucoma, cataracts were detected in $54.5 \%$ of participants; however, only $5.7 \%$ had cataracts that affected visual function (clinically significant cataracts). The following retinal diseases were detected: macular degeneration, $1.2 \%$; diabetic retinopathy, $1.0 \%$; chorioretinal atrophy, $0.5 \%$; macular epiretinal membrane, $2.9 \%$; branch retinal vein occlusion, $0.7 \%$; and others, $2.0 \%$. The use of comprehensive eye examinations including OCT may have contributed to the high prevalence of macular disease. Diabetic retinopathy and macular degeneration are common diseases causing visual impairment in Japan, ${ }^{4,5}$ and the importance of adult ophthalmological checkups for retinal disease screening should be recognized.

Among major diseases causing visual impairment in Japan, glaucoma is an optic nerve disease, and diabetic retinopathy, degenerative myopia, and age-related macular degeneration are retinal diseases. Fundus photography can screen for these diseases simultaneously. ${ }^{23,24}$ Fundus photography is also useful to evaluate the risks of stroke and cardiovascular diseases by examining the retinal blood vessels. $^{25,26}$ Another advantage of fundus photography is that it is noninvasive and can be performed in a short time. Therefore, fundus photography is considered to be suitable for mass screening. However, there are limitations to the use of fundus photography alone for detailed evaluation of the retina and optic nerve, and the risk of overlooking a condition has been indicated. OCT is a diagnostic 
technology that has rapidly developed and spread in the field of ophthalmology and is characterized by its capacity to provide precise tomographic images of the retina and optic nerve noninvasively in a short time. The diagnostic performance of OCT data for detecting glaucoma has been evaluated. $^{27,28}$ Our study suggests that the combination of OCT and fundus photography exerts complementary effects and improves the accuracy of screening for ophthalmic diseases.

The limitations of this study include the fact that the study participants do not reflect the age structure of the Japanese population. The rate of participation in specific health checkups is higher in older people than in younger people. The study participants were those undergoing specific health checkups who also wished to undergo ophthalmological examinations. Therefore, the study participants may have included many unemployed people who had time to spare and people with visual dysfunction or worries about their ophthalmic health. Second, non-contact tonometry, which is not suitable for accurate diagnosis of glaucoma, was used in the study. The equipment for fundus photography and OCT could not be made uniform, although the equipment model and program used for static perimetry were uniform. The diagnosis of ophthalmic diseases was made by each attending physician, and the diagnostic criteria were not completely standardized, whereas the presence of glaucoma was judged by a central committee to ensure standardization and reliability as much as possible. Third, the prevalence and types of glaucoma differ among races and regions. Even within Asia, differences in prevalence according to geographic subregion, type of glaucoma, and habitation have been reported. ${ }^{29,30}$ Lastly, caution may be taken in interpreting odds ratios. In this study, the prevalence of events was $12.4 \%$, and the strength of the association of each risk factor for glaucoma was not very high (1.02-1.85). Therefore, the odds ratio may provide an acceptable approximation of the relative risk. Despite these limitations, the authors believe that the data obtained in this study are of value for predicting the results of checkups in Japan.

In summary, we conducted detailed comprehensive ophthalmic examinations on individuals who visited an ophthalmological medical institution to take the opportunity for specific health checkups. The prevalence of glaucoma was $12.4 \%$, which was higher than the rate previously reported in Japan, suggesting that there are many adults with ocular diseases who should be screened by ophthalmic checkups. The addition of simultaneous ophthalmic checkups to specific health checkups could be an effective measure for the prevention of visual impairment in the older population, although the effectiveness and the cost-effectiveness should be evaluated in the further study.

\section{Acknowledgments}

The following individuals participated in the study. Central unit:

Yoshimune Hiratsuka, Tadashi Nakano, Hiroshi Tamura, Ryo Kawasaki, Shigeru Takano, Tetsuji Yokoyama, and Masakazu Yamada. Study group investigators and clinical sites (all in Japan): Masako Ohtaki (Izumi Eye Clinic, Sendai), Kazushige Sado (Sado Eye Clinic, Sendai), Shinsi Chin (Asahigaoka Eye Clinic, Sendai), Shoko Takano (Tomizawa-Minami Eye Clinic, Sendai), Kazumasa Yamagata (Wakabayashi Eye Clinic, Sendai), Kimiko Fukushita (Karasuyama Eye Clinic, Tokyo), Satoshi Hijikata (Hijikata Eye Clinic, Tokyo), Takami Odajima (Odajima Eye Clinic, Tokyo), Naohisa Nezu (Todoroki Eye Clinic, Tokyo), Hiroshi Katsura (Katsura Eye Clinic, Tokyo), Hiroshi Mochizuki (Mochizuki Eye Clinic, Tokyo), Eri Mikuni (Mikuni Medical \& Eye Clinic, Tokyo), Masaki Shimizu (Shimizu Eye Clinic, Matsue), Sachiko Noda (Noda Eye Clinic, Matsue), Taiji Takanashi (Takanashi Eye Clinic, Matsue), and Ikuko Ichioka (Ichioka Eye Clinic, Matsue).

\section{Author Contributions}

All authors made substantial contributions to conception and design, acquisition of data, or analysis and interpretation of data; took part in drafting the article or revising it critically for important intellectual content; gave final approval of the version to be published; and agree to be accountable for all aspects of the work.

\section{Funding}

This study was supported by a grant (19FA1010) from the Ministry of Health, Labor and Welfare, Japan.

\section{Disclosure}

Professor Masakazu Yamada reports grants from the Ministry of Health, Labor and Welfare, Japan, during the conduct of the study; personal fees from Santen Pharmaceutical Co., Senju Pharmaceutical Co., Otsuka Pharmaceutical Co., and Johnson \& Johnson Vision Care; grants, personal fees from Alcon Japan Co., outside 
the submitted work. Dr Yoshimune Hiratsuka reports grants from the Ministry of Health, Labor and Welfare, Japan, outside the submitted work. Professor Hiroshi Tamura reports grants from Findex, during the conduct of the study; lecture fees from Novartis, outside the submitted work. Professor Ryo Kawasaki reports grants from Ministry of Health, Labor, and Welfare, Japan, during the conduct of the study; is part of the Endowed Department for Topcon, outside the submitted work. Professor Tetsuji Yokoyama reports grants from the Ministry of Health, Labor and Welfare, Japan, during the conduct of the study. Dr Shigeru Takano reports grants from the Ministry of Health, Labor and Welfare, Japan, during the conduct of the study. The authors have no other competing interests, including relevant financial interests, activities, and affiliations in this work.

\section{References}

1. Ko F, Vitale S, Chou C, Cotch M, Saaddine J, Friedman DS Prevalence of nonrefractive visual impairment in US adults and associated risk factors, 1999-2002 and 2005-2008. JAMA. 2012;308(22):2361-2368. doi:10.1001/jama.2012.85685

2. Varma R, Vajaranant TS, Burkemper B, et al. Visual impairment and blindness in adults in the United States: demographic and geographic variations from 2015 to 2050. JAMA Ophthalmol. 2016;134 (7):802-809. doi:10.1001/jamaophthalmol.2016.1284

3. Chou R, Dana T, Bougatsos C, Grusing S, Blazina I. Screening for impaired visual acuity in older adults: updated evidence report and systematic review for the US preventive services task force. JAMA. 2016;315(9):915-933. doi:10.1001/jama.2016.0783

4. Morizane Y, Morimoto N, Fujiwara A, et al. Incidence and cause of visual impairment in Japan: the first nation-wide complete enumeration survey of newly certified visually impaired individuals. Jpn J Ophthalmol. 2019;63(1):26-33. doi:10.1007/s10384-018-0623-4

5. Yamada M, Hiratsuka Y, Roberts CB, et al. Prevalence and causes of visual impairment in adult Japanese population: present status and future projection. Ophthalmic Epidemiol. 2010;17(1):50-57. doi:10.3109/09286580903450346

6. Roberts CB, Hiratsuka Y, Yamada M, et al. Economic cost of visual impairment in Japan. Arch Ophthalmol. 2010;128(6):766-771. doi:10.1001/archophthalmol.2010.86

7. Tamura H, Goto R, Akune Y, Hiratsuka Y, Hiragi S, Yamada M. The clinical effectiveness and cost-effectiveness of screening for age-related macular degeneration in Japan: a Markov modeling study. PLoS One. 2015;10(7):e0133628. doi:10.1371/journal. pone. 0133628

8. Kawasaki R, Akune Y, Hiratsuka Y, Fukuhara S, Yamada M. Costutility analysis of screening for diabetic retinopathy in Japan: a probabilistic Markov modeling study. Ophthalmic Epidemiol. 2015;22(1):4-12. doi:10.3109/09286586.2014.988876

9. Yamada M, Hiratsuka Y, Kawasaki R, et al. Effectiveness and feasibility of vision screening program for adults. The ministry of health labour and welfare, grant-in-aid for scientific research, research report. 2019. Available from: https://mhlw-grants.niph.go.jp/niph/ search/NISR01.do.

10. Andermann A, Blancquaert I, Beauchamp S, Déry V. Revisiting Wilson and Jungner in the genomic age: a review of screening criteria over the past 40 years. Bull World Health Organ. 2008;86 (4):317-319. doi:10.2471/BLT.07.050112
11. Tanihara H, Aihara H, Inatani D, et al. The Japan glaucoma society guidelines for glaucoma (4th edition). Nippon Ganka Gakkai Zasshi. 2018;122(1):5-53.

12. Iwase A, Suzuki Y, Araie M, et al. The prevalence of primary open-angle glaucoma in Japanese: the Tajimi Study. Ophthalmology. 2004;111(9):1641-1648.

13. Yamamoto T, Iwase A, Araie M, et al. The Tajimi Study report 2: prevalence of primary angle closure and secondary glaucoma in a Japanese population. Ophthalmology. 2005;112(10):1661-1669. doi:10.1016/j.ophtha.2005.05.012

14. Suzuki Y, Iwase A, Araie M, et al. Risk factors for open-angle glaucoma in a Japanese population: the Tajimi Study. Ophthalmology. 2006;113 (9):1613-1617. doi:10.1016/j.ophtha.2006.03.059

15. The Ministry of Health, Labor and Welfare, Japan. Overview of implementation of specific health checkups and specific health guidance scheme in 2017 [homepage on the Internet]. 2018. Available from: https://www.mhlw.go.jp/content/12400000/000600883.pdf.

16. Hiratsuka Y, Yokoyama T, Yamada M. Higher participation rate for specific health checkups concerning simultaneous ophthalmic checkups. J Epidemiol. 2020. doi:10.2188/jea.JE20200052

17. Foster PJ. The definition and classification of glaucoma in prevalence surveys. Br J Ophthalmol. 2002;86(2):238-242. doi:10.1136/bjo.86.2.238

18. Anderson DR, Patella VM. Automated Static Perimetry. 2nd ed. St. Louis: Mosby; 1999:121-190.

19. Sawaguchi S, Sakai H, Iwase A, et al. Prevalence of primary angle closure and primary angle-closure glaucoma in a southwestern rural population of Japan: the Kumejima Study. Ophthalmology. 2012;119 (9):1134-1142. doi:10.1016/j.ophtha.2011.12.038

20. Yoshikawa T, Obayashi K, Miyata K, et al. Association between glaucomatous optic disc and depressive symptoms independent of light exposure profiles: a cross-sectional study of the HEIJO-KYO cohort. Br J Ophthalmol. 2019;103(8):1119-1122. doi:10.1136/ bjophthalmol-2018-312176

21. Hatt S, Wormald R, Burr J. Screening for prevention of optic nerve damage due to chronic open angle glaucoma. Cochrane Database Syst Rev. 2006;4:CD006129.

22. Moyer VA. Screening for glaucoma: U.S. preventive services task force recommendation statement. Ann Intern Med. 2013;159 (7):484-489.

23. Myers JS, Fudemberg SJ, Lee D. Evolution of optic nerve photography for glaucoma screening: a review. Clin Exp Ophthalmol. 2018;46(2):169-176. doi:10.1111/ceo.13138

24. Dabasia PL, Fidalgo BR, Edgar DF, Garway-Heath DF, Lawrenson JG. Diagnostic accuracy of technologies for glaucoma case-finding in a community setting. Ophthalmology. 2015;122 (12):2407-2415. doi:10.1016/j.ophtha.2015.08.019

25. Nguyen UT, Bhuiyan A, Park LA, et al. Automated quantification of retinal arteriovenous nicking from colour fundus images. Conf Proc IEEE Eng Med Biol Soc. 2013;2013:5865-5868.

26. Kawasaki R, Xie J, Cheung N, et al. Retinal microvascular signs and risk of stroke: the multi-ethnic study of atherosclerosis (MESA). Stroke. 2012;43(12):3245-3251. doi:10.1161/STROKEAHA.112.673335

27. Virgili G, Michelessi M, Cook J, et al. Diagnostic accuracy of optical coherence tomography for diagnosing glaucoma: secondary analyses of the GATE study. $\mathrm{Br} J$ Ophthalmol. 2018;102(5):604-610. doi:10.1136/bjophthalmol-2017-310642

28. Loewen NA, Zhang X, Tan O, et al. Combining measurements from three anatomical areas for glaucoma diagnosis using Fourier-domain optical coherence tomography. $B r \quad J$ Ophthalmol. 2015;99 (9):1224-1229. doi:10.1136/bjophthalmol-2014-305907

29. Cho HK, Kee C. Population-based glaucoma prevalence studies in Asians. Surv Ophthalmol. 2014;59(4):434-447. doi:10.1016/j. survophthal.2013.09.003

30. Chan EW, Li X, Tham YC, et al. Glaucoma in Asia: regional prevalence variations and future projections. $\mathrm{Br} J$ Ophthalmol. 2016;100(1):78-85. doi:10.1136/bjophthalmol-2014-306102 


\section{Publish your work in this journal}

Clinical Epidemiology is an international, peer-reviewed, open access, online journal focusing on disease and drug epidemiology, identification of risk factors and screening procedures to develop optimal preventative initiatives and programs. Specific topics include: diagnosis, prognosis, treatment, screening, prevention, risk factor modification,

systematic reviews, risk \& safety of medical interventions, epidemiology \& biostatistical methods, and evaluation of guidelines, translational medicine, health policies \& economic evaluations. The manuscript management system is completely online and includes a very quick and fair peer-review system, which is all easy to use. 